\title{
PROGRAMAS DE PÓS-GRADUAÇÃO E PRODUÇÃO DO CONHECIMENTO NO BRASIL: PANORAMA, DESAFIOS E PERSPECTIVAS
}

\author{
JoÃo Ferreira de Olivelra \\ Universidade Federal de Goiás (UFG), Goiânia, Goiás, Brasil \\ MÁrio Luiz NeVEs de AzeVedo \\ Universidade Estadual de Maringá (UEM), Maringá, Paraná, Brasil
}

\begin{abstract}
Resumo: O propósito deste artigo é analisar a situação da produção do conhecimento e da pós-graduação no Brasil, considerando os impactos decorrentes da chamada globalização da economia do conhecimento. Para isto, são examinados dados nacionais e internacionais da produção e difusão do conhecimento. 0 texto realça a nova lógica de regulação e avaliação da pós-graduação no Brasil e destaca que, além dos dilemas econômicos, políticos e sociais existentes, existem desafios que apontam para mudanças significativas na pós-graduação brasileira. O texto também chama atenção para novas tendências na avaliação da pós-graduação e para o necessário diálogo entre coordenações de áreas do conhecimento da CAPES, instâncias de regulação da CAPES e entidades científicas que sempre estiveram presentes e foram protagonistas na constituição do atual sistema de pós-graduação no Brasil.
\end{abstract}

Palavras-chave: Pós-graduação. Produção do conhecimento. Avaliação da Pósgraduação. Economia do conhecimento.

INTRODUÇÃO

Em tempos de globalização e de financeirização da economia, vivencia-se um intenso processo de reestruturação capitalista que tem impactado a educação superior $e$ a produção do conhecimento, provocando, principalmente, uma maior mercadorização da educação superior (graduação e pós-graduação) e da ciência, assim como o chamado produtivismo acadêmico, em detrimento de uma perspectiva de produção do conhecimento pautada em uma maior solidariedade e voltada ao bem público. Essa lógica tem sido justificada, muitas vezes, pela agregação de valor às mercadorias e pela ampliação da competitividade dos países e das empresas em escala global, sobretudo quando vinculadas à geração de tecnologia e inovação.

Nesse contexto, as políticas de financiamento, de avaliação, de gestão universitária, de pesquisa, de pós-graduação e regulação do trabalho acadêmico, dentre outras, evidenciam maior subordinação formal e real da produção do conhecimento aos interesses econômicos de empresas, organizações e agentes nacionais e transnacionais. 
Faz também parte deste processo a pós-graduação, que vem sendo gradativamente reestruturada para assumir novos papéis e finalidades, desenhando-se uma perspectiva de reestruturação de currículos, duração dos cursos e oferta de vagas, novos modos de valorar e aferir a formação discente e impacto da produção, incluindo sobretudo os mestrados e doutorados profissionais no formato presencial ou a distância.

De igual modo, observa-se, dentre outras consequências, a consolidação do conceito de universidade de classe mundial, a adoção de novos mecanismos e processos de avaliação institucional e da produção do conhecimento, assim como o estabelecimento e difusão de rankings universitários, a definição de áreas consideradas estratégicas para a economia e a redução e modelação do financiamento destinado à Ciência, Tecnologia e Inovação (CT\&l) numa perspectiva mais pragmática, gerencialista, produtivista e contábil (LIMA, 1997; SAVIANI, 2010; OLIVEIRA, 2015). É fundamental, pois, refletir sobre a produção do conhecimento em tempos de reestruturação do capitalismo, tendo por base a materialização do processo de globalização da economia do conhecimento.

Há que se questionar, todavia, as finalidades, os sentidos e rumos dessa produção do conhecimento, bem como as mudanças em curso na pós-graduação, na formação e na pesquisa. Esse artigo visa, pois, contribuir com a reflexão sobre essas e outras questões, dado que temos um amplo e consolidado sistema de pós-graduação no Brasil, um modelo de avaliação e fomento da pesquisa e da pós-graduação, que é historicamente dependente de políticas, programas, ações, avaliação e fomento, sobretudo advindos da Coordenação de Aperfeiçoamento de Pessoal de Nível Superior (CAPES) e do Conselho Nacional de Desenvolvimento Científico e Tecnológico (CNPq). Essas duas agências, ambas criadas em 1951, têm historicamente pensado e estabelecido políticas e ações que induzem e regulam a pós-graduação, a pesquisa, as carreiras científicas e a produção e difusão do conhecimento, o que se reflete na posição do Brasil na chamada economia do conhecimento, como veremos no panorama, nos dilemas e desafios atuais.

\section{O PANORAMA DA PÓS-GRADUAÇÃO E DA PRODUÇÃO DO CONHECIMENTO NO BRASIL}

A pós-graduação passou a se estruturar mais efetivamente no Brasil a partir do Parecer no 977/1965, conhecido como Parecer Sucupira. Contudo, com o chamado modelo CAPES de avaliação, instituído a partir de 1976, a pós-graduação ganhou maior importância no contexto das políticas de Estado e reestruturação do campo universitário brasileiro, passando a enfatizar a formação ou qualificação de alto nível e o desenvolvimento da pesquisa, da ciência e da tecnologia. A avaliação e o fomento, por meio da CAPES e do CNPq, tornaram-se pilares fundamentais para a expansão da pósgraduação, para o crescimento do fomento à pesquisa e para o desenvolvimento de carreiras científicas. De modo complementar, foram elaborados, implementados e avaliados, permanentemente, os Planos Nacionais de Pós-graduação (PNPGs), que favoreceram efetivamente a consolidação desse movimento de expansão de cursos, matrículas e titulados, o que contribuiu para a institucionalização da pesquisa e para a elevação da qualidade da formação, sobretudo nas universidades públicas federais e estaduais (OLIVEIRA, 2015; ALVES; OLIVEIRA, 2014). 
As metas 13 e 14 do Plano Nacional de Educação - PNE (2014-2024), de "Elevar a qualidade da educação superior e ampliar a proporção de mestres e doutores do corpo docente em efetivo exercício no conjunto do sistema de educação superior para $75 \%$, sendo, do total, no mínimo, 35\% doutores" e de "Elevar gradualmente o número de matrículas na pós-graduação stricto sensu, de modo a atingir a titulação anual de 60.000 mestres e 25.000 doutores", praticamente já foram alcançadas, como mostram os indicadores mais recentes da Plataforma Sucupira/Capes e do Relatório do $2^{\circ}$ Ciclo de Monitoramento das Metas do Plano Nacional de Educação (PNE) - 2018 (BRASIL. Inep, 2018).

O Brasil conta atualmente com 4.647 programas de pós-graduação ou 7.060 cursos de pós-graduação (Tabela 1). Embora o Mestrado Acadêmico (MA) e o Doutorado Acadêmico (DA) representem o maior percentual, já é significativo o número de programas/cursos de MP (Mestrado Profissional). Os MPs foram regulamentados em 1998 e os DPs, em 2017, por meio da Resolução CNE no 7, de 11 de dezembro de 2017. A legislação atual do Conselho Nacional de Educação (CNE) e da própria CAPES, mediante várias portarias, incentivam a criação de MPs e DPs, sobretudo a distância, por intermédio de Tecnologias Digitais de Informação e Comunicação (TDIC)'. As cinco áreas com maior número de cursos, respectivamente, são: Interdisciplinar (499), ciências Agrárias I (369), Educação (284), Administração Pública e de Empresas, Ciências Contábeis e Turismo (267) e Linguística e Literatura (265).

Tabela 1 - Cursos Avaliados e Reconhecidos de Pós-graduação no Brasil - 2020

\begin{tabular}{|c|c|c|c|c|c|c|c|c|c|c|c|c|}
\hline Item & \multicolumn{7}{|c|}{ Total de Programas de pós-graduação } & \multicolumn{5}{|c|}{ Totais de Cursos de pós-graduação } \\
\hline Nome & Total & ME & DO & MP & DP & $\begin{array}{c}\mathrm{ME} / \\
\mathrm{DO}\end{array}$ & $\begin{array}{c}\mathrm{MP} / \\
\mathrm{DP}\end{array}$ & Total & ME & DO & MP & DP \\
\hline Totais & 4647 & 1337 & 81 & 812 & 4 & 2368 & 45 & 7060 & 3705 & 2449 & 857 & 49 \\
\hline
\end{tabular}

ME: Mestrado Acadêmico DO: Doutorado Acadêmico MP: Mestrado Profissional DP: Doutorado Profissional

ME/DO: Mestrado Acadêmico e Doutorado Acadêmico MP/DP: Mestrado Profissional e Doutorado Profissional

Fonte:

https://sucupira.capes.gov.br/sucupira/public/consultas/coleta/programa/quantitativos/quantitati voAreaAvaliacao.jsf;jsessionid=zP3uyqYKli9mcsi6fMZuCp8X.sucupira-205. Acesso em: 20 mai. 2020

A maior parte dos programas e cursos de pós-graduação encontra-se nas universidades públicas federais e estaduais, espalhadas pelos diferentes estados e regiões do Brasil. Em 2016 havia um total de 4.175, sendo 2.180 (52\%) nas universidades federais, o que tem contribuído para reduzir as desigualdades e assimetrias regionais no Brasil em termos de ensino, pesquisa e extensão (Tabela 2). Os programas mais bem avaliados, com notas 5, 6 e 7, também se encontram nas universidades públicas. A oferta de pósgraduação das IES do setor privado concentra-se nos estados mais ricos, especialmente nas regiões Sul e Sudeste. 
Tabela 02 - Quantitativo de programas de pós-graduação stricto sensu (MS ou DR) em cada um dos Estados e no DF, nos âmbitos federal, estadual, municipal e privado - 2016

\begin{tabular}{|c|c|c|c|c|c|c|c|}
\hline Estado & $\begin{array}{c}\text { Univ. } \\
\text { Federais }\end{array}$ & $\begin{array}{l}\text { Outras } \\
\text { IES } \\
\text { Federais }\end{array}$ & Estaduais & Municipais & Privadas & $\begin{array}{c}\text { Total } \\
\text { (b) }\end{array}$ & $\begin{array}{c}\% \\
(\mathrm{a} / \mathrm{b})\end{array}$ \\
\hline Rondônia & 12 & - & - & - & - & 12 & 100 \\
\hline Acre & 10 & - & - & - & - & 10 & 100 \\
\hline Amazonas & 36 & 11 & 9 & - & 2 & 58 & 62 \\
\hline Roraima & 10 & - & 4 & - & - & 14 & 71 \\
\hline Pará & 85 & 5 & 9 & - & 8 & 107 & 79 \\
\hline Amapá & 4 & - & - & - & - & 4 & 100 \\
\hline Tocantins & 22 & - & - & - & - & 22 & 100 \\
\hline Maranhão & 29 & 2 & 11 & - & 4 & 46 & 63 \\
\hline Piauí & 36 & 1 & 2 & - & 1 & 40 & 90 \\
\hline Ceará & 69 & 6 & 32 & - & 15 & 122 & 57 \\
\hline Rio Gr. do Norte & 81 & 2 & 14 & - & 7 & 104 & 78 \\
\hline Paraíba & 87 & 1 & 16 & - & 2 & 106 & 82 \\
\hline Pernambuco & 123 & 4 & 15 & - & 19 & 161 & 76 \\
\hline Alagoas & 35 & - & 1 & - & 3 & 39 & 90 \\
\hline Sergipe & 44 & 1 & - & - & 5 & 50 & 88 \\
\hline Bahia & 91 & 4 & 66 & - & 17 & 178 & 51 \\
\hline Minas Gerais & 320 & 27 & 16 & - & 60 & 423 & 76 \\
\hline Espírito Santo & 55 & 6 & - & - & 13 & 74 & 74 \\
\hline Rio de Janeiro & 242 & 79 & 73 & - & 85 & 479 & 51 \\
\hline São Paulo & 131 & 17 & 492 & 16 & 243 & 899 & 15 \\
\hline Paraná & 124 & 1 & 149 & - & 52 & 326 & 38 \\
\hline Santa Catarina & 79 & 2 & 30 & 15 & 41 & 167 & 47 \\
\hline Rio Gr. do Sul & 207 & 32 & - & - & 155 & 394 & 53 \\
\hline Mato G. do Sul & 51 & - & 10 & - & 7 & 68 & 75 \\
\hline Mato Grosso & 38 & 1 & 11 & - & 7 & 57 & 67 \\
\hline Goiás & 66 & 11 & 10 & 1 & 14 & 102 & 65 \\
\hline Distrito Federal & 93 & 4 & 1 & - & 15 & 113 & 82 \\
\hline Total Geral & 2.180 & 217 & 971 & 32 & 775 & 4.175 & 52 \\
\hline
\end{tabular}

Fonte: Amaral \& Oliveira (2020). Elaborado a partir de dados do GeoCapes (2019).

As matrículas na pós-graduação stricto sensu vêm aumentando significativamente, como mostra a Tabela 3 , a seguir, que traz o número de matrículas no período de 2015 a 2018. Há certa irregularidade nas matrículas, o que pode ser observado na queda de 2016 em relação a 2015 no tocante às matrículas nas instituições de ensino superior (IES) privadas, e de 2018 em relação a 2017, tanto nas matrículas privadas como 
nas públicas. Isso parece refletir a crise vivenciada pelo país e pelas agências de fomento, sobretudo Capes e CNPq, atingido as bolsas de estudo e as IES, sobretudo as universidades públicas. Quando se compara os períodos de 2015 e 2018 a queda das matrículas ocorre em todas as esferas.

Tabela 3 - Número de matrículas da Pós-graduação stricto sensu no período 2015 a 2018

\begin{tabular}{|c|c|c|c|c|c|c|}
\hline \multirow{3}{*}{ Ano } & \multirow{3}{*}{$\begin{array}{l}\text { Total } \\
\text { Geral }\end{array}$} & \multicolumn{5}{|c|}{ Categoria Administrativa } \\
\hline & & \multicolumn{4}{|c|}{ Pública } & \multirow{2}{*}{ Privada } \\
\hline & & Total & Federal & Estadual & Municipal & \\
\hline 2015 & 325.230 & 269.395 & 184.041 & 83.856 & 1.498 & 55.835 \\
\hline 2016 & 347.035 & 292.023 & 200.442 & 89.937 & 1.644 & 55.012 \\
\hline 2017 & 361.530 & 304.146 & 210.305 & 92.217 & 1.624 & 57.384 \\
\hline 2018 & 288.590 & 245.131 & 170.803 & 73.048 & 1.280 & 43.459 \\
\hline \multicolumn{2}{|c|}{$\Delta \% 2018 / 2015$} & $-0,09$ & $-0,07$ & $-0,13$ & $-0,15$ & $-0,22$ \\
\hline
\end{tabular}

Fonte: Censos da Educação Superior do Inep

O número de títulos de mestrado acadêmico (MA) e profissional (MP) concedidos no Brasil no período de 2010-2018 teve aumento constante (Tabela 4), ultrapassando a meta do PNE (2014-2024). O crescimento foi maior, no entanto, no MP. Quando se observa o crescimento no número de títulos de mestrado (MA e MP) por região no mesmo período vemos que as regiões Norte, Nordeste e Centro-Oeste registraram maior crescimento (Tabela 5).

Tabela 4 - Número de títulos de mestrado concedidos- Brasil - 2010-2018

\begin{tabular}{|l|r|r|r|r|r|r|r|r|r|}
\hline Mestrado & $\mathbf{2 0 1 0}$ & $\mathbf{2 0 1 1}$ & $\mathbf{2 0 1 2}$ & $\mathbf{2 0 1 3}$ & $\mathbf{2 0 1 4}$ & $\mathbf{2 0 1 5}$ & $\mathbf{2 0 1 6}$ & $\mathbf{2 0 1 7}$ & $\mathbf{2 0 1 8}$ \\
\hline MA & 36.247 & 39.544 & 42.878 & 45.490 & 46.245 & 47.644 & 49.002 & 50.306 & 51.610 \\
\hline MP & 3.343 & 3.689 & 4.260 & 6.045 & 6.967 & 9.023 & 10.612 & 10.841 & 12.822 \\
\hline Total & 39.590 & 43.233 & 47.138 & 51.535 & 53.212 & 56.667 & 59.614 & 61.147 & 64.432 \\
\hline
\end{tabular}

Fonte: Dados levantados no âmbito do Projeto Integrado de Pesquisa "Expansão e qualidade da educação superior no contexto do Plano Nacional de Educação (2014-2024): Tensões, limites e perspectivas", UFG/UFRN, financiado pelo CNPq. 
Tabela 5 - Número de títulos de mestrado concedidos, por grande região - Brasil 2010-2018

\begin{tabular}{|l|r|r|r|r|r|r|r|r|r|}
\hline \multicolumn{1}{|c|}{ Item } & $\mathbf{2 0 1 0}$ & $\mathbf{2 0 1 1}$ & $\mathbf{2 0 1 2}$ & $\mathbf{2 0 1 3}$ & $\mathbf{2 0 1 4}$ & $\mathbf{2 0 1 5}$ & $\mathbf{2 0 1 6}$ & $\mathbf{2 0 1 7}$ & $\mathbf{2 0 1 8}$ \\
\hline Brasil & 39.590 & 43.233 & 47.138 & 51.535 & 53.212 & 56.667 & 59.614 & 61.147 & 64.432 \\
\hline Norte & 1.407 & 1.587 & 2.050 & 2.020 & 2.126 & 2.448 & 2.728 & 2.887 & 3.334 \\
\hline Nordeste & 6.750 & 7.505 & 8.301 & 9.605 & 10.090 & 10.597 & 10.863 & 11.328 & 12.630 \\
\hline Sudeste & 20.800 & 22.170 & 23.480 & 25.211 & 25.694 & 27.193 & 28.791 & 28.874 & 29.762 \\
\hline Sul & 8.103 & 8.902 & 9.949 & 10.756 & 11.046 & 11.972 & 12.450 & 13.312 & 13.595 \\
\hline C. Oeste & 2.530 & 3.069 & 3.358 & 3.943 & 4.256 & 4.457 & 4.782 & 4.746 & 5.111 \\
\hline
\end{tabular}

Fonte: Dados levantados no âmbito do Projeto Integrado de Pesquisa "Expansão e qualidade da educação superior no contexto do Plano Nacional de Educação (2014-2024): Tensões, limites e perspectivas", UFG/UFRN, financiado pelo CNPq.

Observa-se também aumento significativo na titulação de doutores (2010 a 2018), chegando perto de alcançar a titulação prevista na meta 14 do PNE (2014-2024) de atingir a titulação anual de 25.000 doutores ano (Tabela 6). O número de doutores no Brasil por habilitantes no país, no entanto, é considerado baixo se comparado aos países ricos e com desenvolvimento tecnológico em várias fronteiras do conhecimento.

Tabela 6 - Número de títulos de doutorado concedidos- Brasil - 2007-2018

\begin{tabular}{|l|r|r|r|r|r|r|r|r|r|}
\hline Ano & 2010 & 2011 & 2012 & 2013 & 2014 & 2015 & 2016 & 2017 & 2018 \\
\hline Doutorado & 11.314 & 12.321 & 13.912 & 15.650 & 17.286 & 18.996 & 20.603 & 21.591 & 22.894 \\
\hline
\end{tabular}

Fonte: Dados levantados no âmbito do Projeto Integrado de Pesquisa "Expansão e qualidade da educação superior no contexto do Plano Nacional de Educação (2014-2024): Tensões, limites e perspectivas", UFG/UFRN, financiado pelo CNPq.

Todo esse crescimento da pós-graduação tem resultado em um impressionante aumento da produtividade científica no Brasil. Segundo análise do Grupo Web of Science, o Brasil ocupa a $13^{a}$ posição no mundo em termos de produção de artigos e revisões de pesquisa indexados (Figura 1). As universidades públicas constituem-se no principal locus da pesquisa científica e na principal fonte de publicações no Brasil. O estudo mostra ainda a ampliação da colaboração internacional dos pesquisadores brasileiros, que já chega a 205 países. Mostra também, no tocante ao impacto da citação, que "A porcentagem de papéis [artigos] brasileiros no primeiro por cento dos artigos mais citados do mundo é consistentemente próximo a um por cento durante o período de seis anos, 2013-2018" (WEB OF SCIENCE GROUP, 2019, p. 2), ou seja, ainda é baixo, mas está melhorando constantemente. 
Figura 1 - Artigos e Revisões indexados na Web of Science, 2013 - 2018

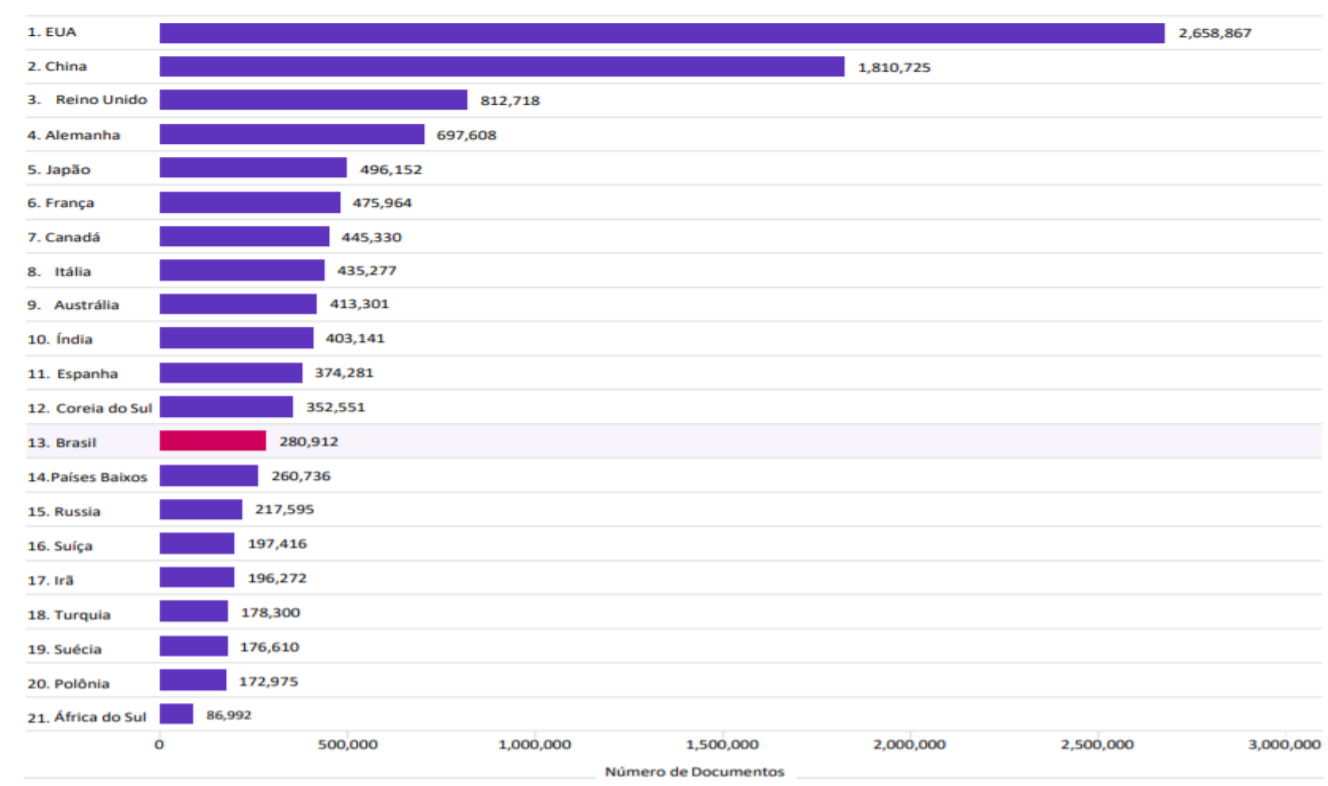

Fonte: Clarivate Analytics. Grupo Web of Science (2019).

O estudo do Grupo Web of Science também mostra que, na comparação entre pares, estamos na média da produção dos países de economias emergentes - BRICS (Brasil, Rússia, Índia, China e África do Sul) no período de 2013 a 2018. A colaboração com indústrias, estratégia "para promover colaborações de pesquisa entre a universidade e a indústria estão dando frutos em termos do número de artigos publicados", embora ainda seja considerada baixa a conexão com a inovação tecnológica e social. De modo geral, mesmo mostrando o crescimento da produção do conhecimento no Brasil, o estudo enfatiza a "importância das colaborações internacionais e com a indústria na visibilidade e no impacto da pesquisa" (WEB OF SCIENCE GROUP, 2019).

A Tabela 7, a seguir, expõe o número de artigos de cientistas \& engenheiros em todos os campos, por país ou economia, no período de 2006 e 2016, tendo por base os 14 países mais bem classificados. A China e os EUA ocupam as duas primeiras posições, sendo que a China ultrapassou os EUA quando são comparados os dados de 2006 e 2016. "Um terço do ganho mundial de 2006 a 2016 refletiu um crescimento no número de artigos da China. No entanto, as publicações dos EUA receberam mais citações do que as publicações da China" (NSF, 2017). Na sequência, os países melhores classificados em 2006 foram: Japão, Reino Unido e Alemanha. Mas, em 2016, aparecem Índia, Alemanha e Reino Unido. O Brasil aparece na 12a posição em 2016 com 53.607 artigos. 
Tabela 7 - Artigos de Cientistas \& Engenheiros em todos os campos, por país ou economia: 2006 e 2016

\begin{tabular}{|c|c|c|c|c|c|c|c|}
\hline Clas. & $\begin{array}{l}\text { País ou } \\
\text { economia }\end{array}$ & $\begin{array}{l}\text { Status econômico } \\
\text { do país ou da } \\
\text { economia }\end{array}$ & 2006 & 2016 & $\begin{array}{c}\text { Variação } \\
\text { média } \\
\text { anual }(\%)\end{array}$ & $\begin{array}{c}\text { Total } \\
\text { mundial } \\
\text { em } 2016 \\
(\%)\end{array}$ & $\begin{array}{c}\text { Total } \\
\text { acumulado } \\
\text { no mundo } \\
\text { em } 2016(\%)\end{array}$ \\
\hline - & Mundo & $\mathrm{n} / \mathrm{D}$ & 1.567 .422 & 2.295 .608 & 3.9 & $\mathrm{n} / \mathrm{D}$ & $\mathrm{n} / \mathrm{D}$ \\
\hline 1 & China & $\begin{array}{l}\text { Em } \\
\text { desenvolvimento }\end{array}$ & 189.760 & 426.165 & 8.4 & 18,6 & 18,6 \\
\hline 2 & EUA & Desenvolvido & 383.115 & 408.985 & 0,7 & 17,8 & 36,4 \\
\hline 3 & Índia & $\begin{array}{l}\text { Em } \\
\text { desenvolvimento }\end{array}$ & 38.590 & 110.320 & 11.1 & 4.8 & 41,2 \\
\hline 4 & Alemanha & Desenvolvido & 84.434 & 103.122 & 2.0 & 4.5 & 45,7 \\
\hline 5 & $\begin{array}{l}\text { Reino } \\
\text { Unido }\end{array}$ & Desenvolvido & 88.061 & 97.527 & 1.0 & 4.3. & 50,0 \\
\hline 6 & Japão & Desenvolvido & 110.503 & 96.536 & $-1,3$ & 4.2 & 54,2 \\
\hline 7 & França & Desenvolvido & 62.448 & 69.431 & 1.1 & 3.0 & 57,2 \\
\hline 8 & Itália & Desenvolvido & 50.159 & 69.125 & 3.3 . & 3.0 & 60,3 \\
\hline 9 & $\begin{array}{l}\text { Coreia do } \\
\text { Sul }\end{array}$ & Desenvolvido & 36.747 & 63.063 & 5.5 & 2.8 & 63,0 \\
\hline 10 & Rússia & $\begin{array}{l}\text { Em } \\
\text { desenvolvimento }\end{array}$ & 29.369 & 59.134 & 7.2 & 2.6 & 65,6 \\
\hline 11 & Canadá & Desenvolvido & 49.259 & 57.356 & 1.5 & 2.5 & 68,1 \\
\hline 12 & Brasil & $\begin{array}{l}\text { Em } \\
\text { desenvolvimento }\end{array}$ & 28.160 & 53.607 & 6.6. & 2.3 & 70,4 \\
\hline 13 & Espanha & Desenvolvido & 39.271 & 52.821 & 3.0 & 2.3 & 72,7 \\
\hline 14 & Austrália & Desenvolvido & 33.100 & 51.068 & 4.4 . & 2.2 & 75,0 \\
\hline
\end{tabular}

Fontes: Fundação Nacional de Ciência, Centro Nacional de Estatísticas de Ciência e Engenharia; SRI Internacional; Science-Metrix; Elsevier, Scopus abstract and citation database, acessado em julho de 2017.

Disponível em: https:/www.nsf.gov/statistics/2018/nsb20181/report/sections/academic-researchand-development/outputs-of-s-e-research-publications\#publication-output-by-country. Acesso em $20 / 05 / 2020$

Um dos grandes problemas apontados nos estudos sobre a produção científica no Brasil refere-se ao registro de patentes. A Tabela 8 , a seguir, mostra os pedidos e decisões de propriedade industrial de 2014 a 2019, conforme dados do Instituto Nacional da Propriedade Industrial (INPI). Embora baixo, se comparado aos países desenvolvidos, o Brasil evidencia que vem decrescendo o depósito de patentes de 2014 a 2018, com pequena recuperação em 2019. As decisões sobre concessões, no entanto, evidenciam um crescimento constante e acentuado, sobretudo de 2017 a 2019. É considerável também o crescimento dos depósitos e concessões de marcas e menos expressivo o 
OLIVEIRA, J. F. de $;$ AZEVEDO, M. L. N. de

depósito e concessões de desenhos industriais, programas de computador, contratos de tecnologia, indicações geográficas e topografias de circuito integrado (INPI, 2020, p. 16).

Tabela 8 - Pedidos e Decisões de Propriedade Industrial (Patentes) - 2014 a 2019

\begin{tabular}{|l|r|r|r|r|r|r|}
\hline \multicolumn{1}{|c|}{ Patentes } & \multicolumn{1}{c|}{$\mathbf{2 0 1 4}$} & \multicolumn{1}{c|}{$\mathbf{2 0 1 5}$} & \multicolumn{1}{c|}{$\mathbf{2 0 1 6}$} & \multicolumn{1}{c|}{$\mathbf{2 0 1 7}$} & \multicolumn{1}{c|}{$\mathbf{2 0 1 8}$} & \multicolumn{1}{c|}{$\mathbf{2 0 1 9}$} \\
\hline Depósitos & 33.182 & 33.043 & 31.020 & 28.667 & 27.551 & 28.318 \\
\hline Decisões & 22.336 & 15.842 & 25.481 & 44.781 & 41.503 & 44.486 \\
\hline Concessões & 3.123 & 3.895 & 4.771 & 6.250 & 11.090 & 13.750 \\
\hline Indeferimentos & 2.586 & 2.864 & 3.167 & 4.324 & 5.701 & 5.270 \\
\hline Arquivamentos & 16.574 & 8.978 & 17.488 & 34.112 & 24.637 & 25.340 \\
\hline $\begin{array}{l}\text { Desistências } \\
\text { Homologadas }\end{array}$ & 53 & 105 & 55 & 95 & 75 & 126 \\
\hline
\end{tabular}

Fonte: Elaborada a partir de dados do INPI (2020, p.2)

\section{DILEMAS E DESAFIOS ATUAIS NA PRODUÇÃO DO CONHECIMENTO E NA PÓS-GRADUAÇÃO}

Mesmo que os números da produção científica e da pós-graduação brasileiras, em termos absolutos, pareçam elevados proporcionalmente, em comparação com a população e levando em consideração as necessidades tecnológicas, de conhecimento novo e de formação de pessoal com pós-graduação, inclusive docentes para a educação básica, muito ainda tem de se avançar. Marginson (2018), em longo estudo sobre "A Nova Geopolítica da Educação Superior", mostra que o mapa da produção científica global está mudando, com a China ganhando cada vez mais participação no mundo da ciência.

Figura 2 - Número anual de artigos científicos publicados, 2003-2016 Estados Unidos, China, Alemanha, Reino Unido, Coréia do Sul

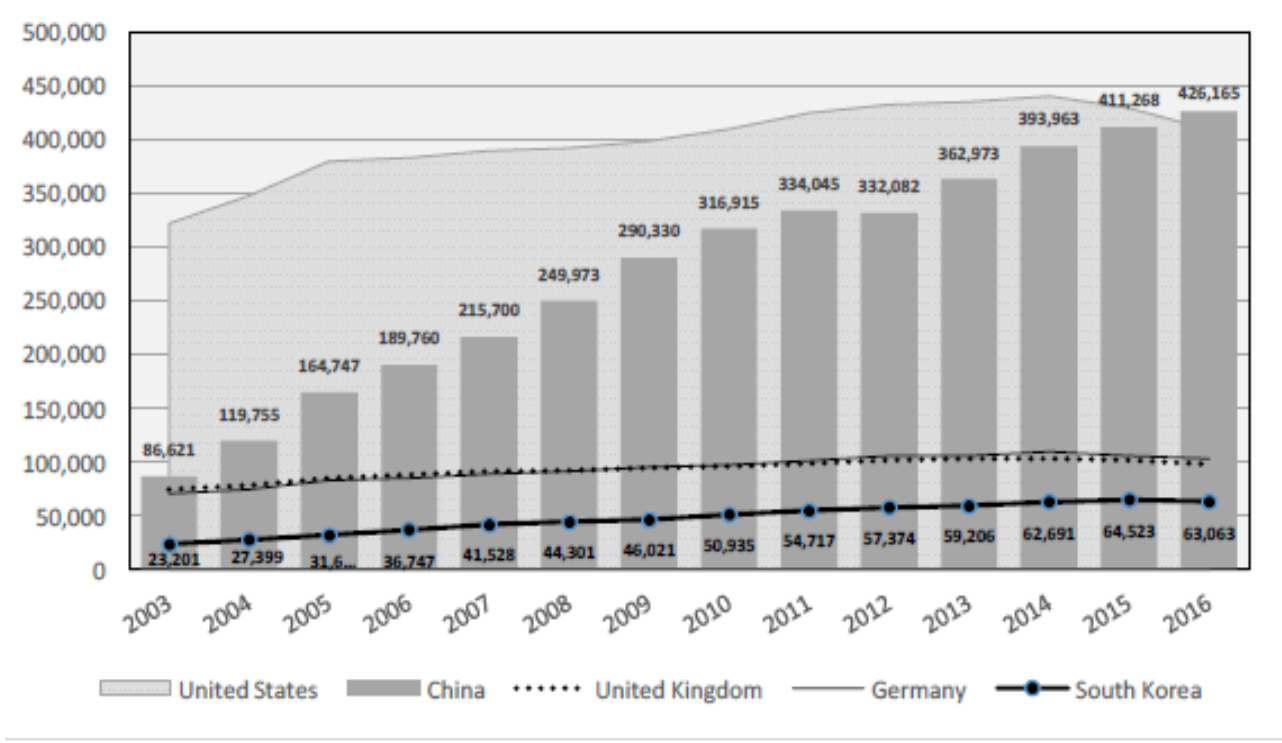

Fonte: Marginson, 2018, p. 42. 
É preciso também ressaltar, conforme alertam Azevedo, Oliveira e Catani (2016) que, "apesar do avanço do Brasil na produção científica mundial, a massa crítica acadêmica encontra-se nos países centrais, não por acaso os mesmos espaços de concentração de poder, tecnologia e riqueza" (p. 789). Do ponto de vista da produção econômica, se o mundo pudesse ser representado como uma bola de futebol, os gomos do "capotão" espelhariam a distribuição do Produto Interno Bruto por país e por continente no Globo (notem que o México, mesmo estando situado na América do Norte, nesta "bola" está na América Latina ao lado do Brasil).

Figura 3 - Participação Regional/Continental na Produção Global

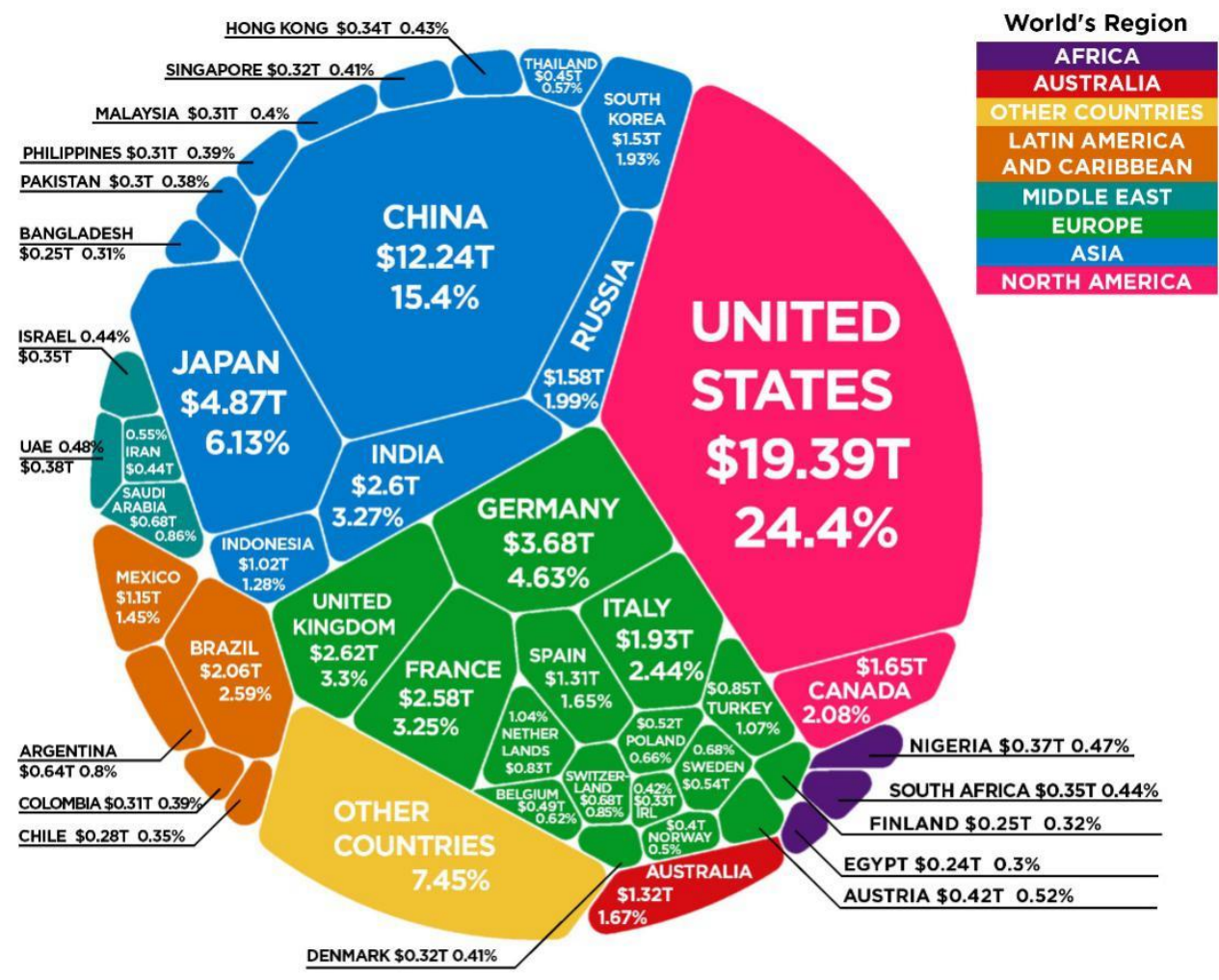

Article and Sources:

https://howmuch.net/articles/the-world-economy-2017

howmuch net $^{\text {now }}$

Fonte: howmuch.net - Understanding Money, 2017

Nesse contexto, é necessário ressaltar que o Novo Regime Fiscal (NRF), aprovado em 2016, no Governo de Michel Temer (2016-2018), especialmente por meio da Emenda Constitucional no 95/2016, é um fator que entrava o cumprimento das 20 metas do PNE 
(2014-2024) e embaraça a sorte de o Brasil conquistar capacidade de produção científica em fronteiras do conhecimento e em áreas complexas que são necessárias para o desenvolvimento do País, pois, conforme Azevedo (2016a),

o NRF é regressivo e inibe o lançamento e a sustentabilidade de políticas públicas de promoção de justiça social, de solidariedade e de desenvolvimento do País, inclusive por intermédio de políticas para o avanço da Ciência, Tecnologia e Inovação, pois congela as despesas primárias e permite arrastar ainda mais recursos para os que têm mais, a exemplo dos detentores da "dívida pública" (p. 257).

Ademais, as políticas públicas para a pós-graduação no Brasil recebem modulação, conforme alertam Ball e Bowe (1992), do contexto da influência, em escalas regional, nacional e global. Assim, por um lado, atores sociais que operam em escalas estaduais, regionais e nacional, mas que também estão conectados com atores internacionais, têm interesses e procuram persuadir as instâncias da CAPES e do Ministério da Educação (MEC) na moldagem das políticas para o SNPG. Por outro lado, levando em conta a escala global, os formuladores da regulação da pós-graduação no Brasil têm considerado que o mundo está passando pela quarta revolução tecnológica (SCHWAB, 2016), também chamada de sociedade ou economia baseada no conhecimento. De acordo com Azevedo (2013),

\begin{abstract}
A economia baseada no conhecimento não é exatamente uma mudança de paradigma em que se saltaria para uma economia baseado em serviços e na produção de bens de alto valor agregado: tem sido, na realidade, uma ideologia de inculcação do habitus individualista, da responsabilização do trabalhador por sua empregabilidade, da mercadorização da oferta da educação e do "cercamento" do conhecimento (AZEVEDO, 2013, p. 109-110).
\end{abstract}

O SNPG, conforme dados anteriormente citados, apesar de estar bem estabelecido, ainda apresenta fragilidades, dilemas e desafios. Assim, por demanda do Conselho Superior da CAPES, a "Comissão Especial de Acompanhamento do PNPG 20112020" (daqui em diante, denominada "Comissão 2020"2) apresentou, em fevereiro de 2020, Relatório de seus trabalhos, contendo a "Proposta de Aprimoramento da Avaliação da Pós-Graduação Brasileira para o Quadriênio 2021-2024 - Modelo Multidimensional".

O relatório da Comissão 2020, na Introdução, reconhece que "o Sistema Nacional de Pós-graduação (SNPG) consolidou-se ao longo das últimas décadas ganhando visibilidade nacional e internacional" (BRASIL. CAPES, 2020, p. 2). Entretanto, referenciando-se no novo Marco Legal de Ciência, Tecnologia e Inovação, que teve por base, especialmente, a Emenda Constitucional no 85 , de 26 de fevereiro de 2015 , que trata sobre atividades de ciência, tecnologia e inovação, a Comissão 2020 afirma que as novas regras do Marco Legal de C,T \& I favorecem "a criação de um ambiente de inovação mais dinâmico no Brasil" (BRASIL. CAPES, 2020, p. 6) e que a "Capes terá um papel particularmente importante ao valorizar, via sistema de avaliação, os Programas voltados à inovação, à transferência de conhecimento e ao impacto na sociedade" (BRASIL. CAPES, 2020, p. 7). Neste sentido, a Comissão 2020 propõe, para a avaliação dos programas de pós-graduação, um "modelo multidimensional" composto por cinco dimensões: 1) 
Formação de Pessoal; 2) Pesquisa; 3) Inovação e Transferência de Conhecimento; 4) Impacto na Sociedade; 5) Internacionalização (BRASIL. CAPES, 2020, p. 8).

Não é segredo que o modelo multidimensional proposto pela Comissão 2020 tem por referência o projeto U-Multirank, financiado pela Comissão Europeia. O portal da Capes fez esse anúncio público, ainda em 2019, reconhecendo que o modelo multidimensional "é inspirado no sistema europeu de avaliação das instituições, o U-Multirank" (BRASIL. CAPES, 02 out. 2019). De acordo com os organizadores do projeto U-Multirank europeu, "baseado em resultados de um estudo de viabilidade de 150 universidades realizado em 2010/2011, a Comissão Europeia decidiu financiar a implementação do U-Multirank"33 (U-MULTIRANK, 15 jun. 2020). Para evidenciar o contexto da influência externa na formatação de políticas públicas, conforme alertam Ball e Bowe (1992), pode-se notar que as dimensões de avaliação do projeto original europeu, declaradamente orientadas para compor tabelas de classificação internacional (rankings), são bastante próximas do modelo multidimensional que vem tramitando na Capes:

O U-Multirank é uma abordagem multidimensional e é orientada ao usuário para composição de ranking internacional de instituições de educação superior. Ela [a plataforma U-Multirank] compara a performance de instituições de educação superior - as universidades - nas cinco dimensões da atividade universitária: (1) ensino e aprendizagem, (2) pesquisa, (3) transferência de conhecimento, (4) orientação internacional [internacionalização] e (5) [pertinência] compromisso regional (U-MULTIRANK, 15 jun 2020).

O modelo de avaliação dos programas de pós-graduação, vigente para o quadriênio (2017-2021), baseia-se em "um modelo único, com uma escala nacional e com padrões e critérios que em princípio são uniformes, mas que sofrem pequenas adaptações para atender as especificidades das diferentes áreas" (BRASIL. CAPES, 2020, p. 7). Para a área da Educação (uma das 49 áreas de conhecimento para fins de avaliação da (APES), os critérios de avaliação constantes na ficha de avaliação, proposta pela Coordenação de Área de Educação e aprovada pelo Conselho Técnico-Científico (CTC) da CAPES, válida para o quadriênio 2016-2021, são três os quesitos a serem avaliados, distribuídos em doze itens:

Quadro 1 - Quesitos e itens de avaliação constantes na ficha de avaliação da área de Educação da CAPES

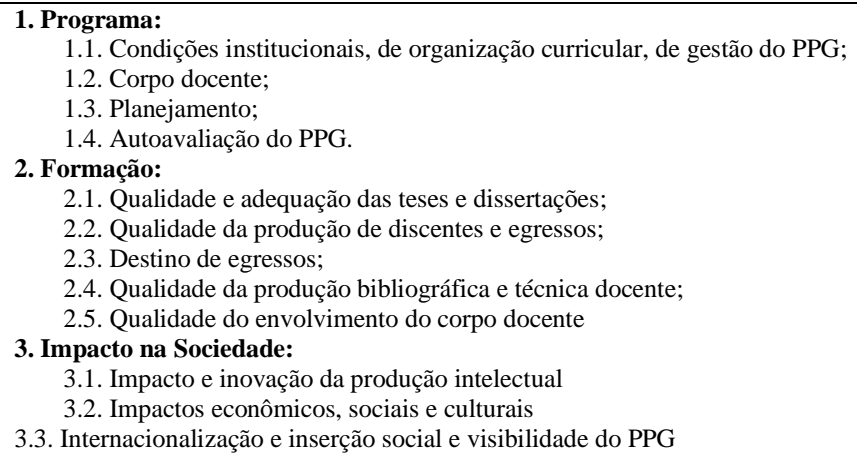

Fonte: BRASIL. CAPES. Ficha de Avaliação - 38 Educação - 2017/2020 
Assim, de maneira sumária, baseados nas informações prestadas pela Coordenação de Área de Educação e na própria ficha de avaliação, percebe-se que a nota final dos Programas de Pós-graduação em Educação (PPGEs) tem sido formada por indicadores qualitativos, representando $66 \%$ da avaliação, e por indicadores quantitativos (pouco mais de $1 / 3$ da nota final, ou 34\%). Para melhor compreensão, vale elencá-los no Quadro 2:

Quadro 2 - Indicadores qualitativos e quantitativos da ficha de avaliação dos PPG elaborada pela Capes para o quadriênio 2017 a $2020^{5}$

\begin{tabular}{|c|c|}
\hline $\begin{array}{c}\text { Indicadores Qualitativos } \\
\text { (aproximadamente } 66 \% \text { da nota) }\end{array}$ & $\begin{array}{l}\text { Indicadores Quantitativos } \\
\text { (aproximadamente 34\% da nota) }\end{array}$ \\
\hline 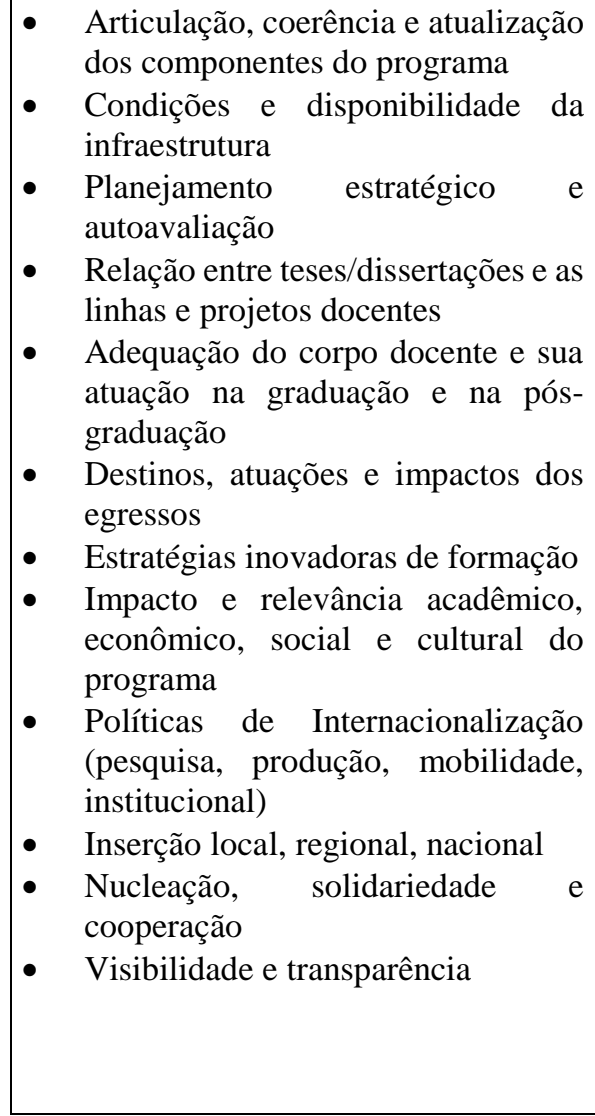 & $\begin{array}{l}\text { - } \% \text { de trabalhos de conclusão que } \\
\text { - } \% \text { de bolsistas de mestrado que defenderam } \\
\text { em } 30 \text { meses e de doutorado em } 48 \text { meses } \\
\text { - } \% \text { de discentes-autores } \\
\text { - } \% \text { de egressos-autores } \\
\text { com de discentes-autores e egressos-autores } \\
\text { qualificados em periódicos bem } \\
\text { - \% de egressos-autores com artigos em } \\
\text { periódicos bem qualificados } \\
\text { - dos DP cujos projetos contam com } \\
\text { participação discente } \\
\text { - dos DP que realizam pesquisa, } \\
\text { orientação e docência } \\
\text { - } \% \text { dos DP publicam em coautoria com } \\
\text { discentes ou egressos } \\
\text { - de atividades da PG sob } \\
\text { responsabilidade dos DP } \\
\text { \% de bolsistas de mestrado que defenderam } \\
\text { em } 30 \text { meses e de doutorado em } 48 \text { meses } \\
\text { em programas acadêmicos } \\
\text { - } \% \text { dos DP que publicaram artigos A1 ou } \\
\text { Livro L1 ou L2 } \\
\text { Razão artigos A1+A2 e total dos DP } \\
\text { Média ponderada da produção } \\
\text { bibliográfica docente }\end{array}$ \\
\hline
\end{tabular}

Fonte: BRASIL. CAPES. Ficha de Avaliação - 38 Educação - 2017/2020

Dessa forma, a nota final dos programas de pós-graduação é a composição proporcional de narrativas qualitativas (66\%), que interpretam a relevância de cada curso na formação de mestres e doutores e seu impacto na sociedade, e de indicadores quantitativos (34\%) referentes, fundamentalmente, à rotina de pesquisas docente e discente (produção científica). Para a avaliação dos PPGEs na área de Educação, no quadriênio 2017-2020, serão indicados até quatro textos por docente de cada Programa ${ }^{6}$. 
Vale ressaltar que, para o próximo quadriênio (2021-2024), a Comissão 2020 sugere "manter a atual escala de notas de 1 a 7 , já consolidada na comunidade" (BRASIL. CAPES, 2020, p. 25).

Vemos, portanto, que estão previstas alterações significativas na avaliação dos programas/cursos de pós-graduação e que, no próximo quadriênio (2021-2024), se adotado o modelo "Proposta de Aprimoramento da Avaliação da Pós-Graduação Brasileira para o Quadriênio 2021-2024 - Modelo Multidimensional", haverá mudança de paradigma no modelo CAPES de avaliação da pós-graduação brasileira que, em boa medida, se ajusta ao proposto em Resolução CNE/CES no 7/2017do CNE, Portaria MEC no 389/2017 e Portaria CAPES no 131/2017, que dispõem sobre os mestrados e doutorados (M/D) profissionais e, ainda, a Portaria no 90/2019, que dispõe sobre os programas de pósgraduação stricto sensu na modalidade de educação a distância, e Portaria nº 70/2020, que regulamenta a criação de polos de educação a distância para cursos de pósgraduação stricto sensu. De modo que, conforme análise de Oliveira e Lima (2018, p.237),

A lógica, os fins e o modus operandi do sistema de pós-graduação está sendo fortemente alterada, como evidencia a nova base legal. Dentre as alterações em curso podem ser destacadas:

a) processo de reconfiguração da pós-graduação pautado numa (des)regulamentação que favorece maior flexibilização dos parâmetros de oferta e maior articulação com os interesses de mercado, especialmente para expansão privada dos M/D profissionais.

b) alterações nos fins da pós-graduação, antes centrados na formação de pesquisadores, de docentes para magistério superior e de profissionais de alto nível intelectual, que começam a migrar para uma formação centrada no desenvolvimento de competências técnico-profissional, na geração de tecnologias e inovações e na formação continuada de professores;

c) ênfase na expansão da oferta de $M / D$ profissionais via $E a D$ em instituições privadas com uso intensivo da EaD;

d) redução do financiamento para pesquisa e manutenção dos programas de pós-graduação nas IES públicas, em razão da crise econômica e das novas prioridades da Capes;

e) adoção de novos critérios, mecanismos e processos de avaliação, ajustados aos parâmetros internacionais ou às demandas por expansão de cursos profissionais;

f) implementação de mestrado profissional via EaD pelas instituições públicas, com certa ampliação da oferta de cursos e diminuição dos recursos e condições de oferta. (OLIVEIRA: LIMA, 2018, p. 237).

Em resposta ao relatório da Comissão 2020, os três Colégios que organizam as 49 Áreas de conhecimento e avaliação da própria Capes produziram Carta Aberta, com data de 16 de junho de 2020, em que afirmam que o referido relatório ignorou "algumas alterações já implantadas e testadas no Seminário de Meio Termo, realizado em 2019, e pautadas em documentos chancelados pelas instâncias competentes da CAPES e disponibilizados em página específica". Por conseguinte, questionam a ausência de 
debate com a comunidade científica que compõe a CAPES, sobretudo em relação aos seguintes pontos: Redefinição das áreas de conhecimento; Avaliação de impacto (definição de indicadores e avaliação por comissão externa); Indicadores únicos para todas as áreas; Extinção do Qualis periódicos; Resultados financeiros como indicador de impacto; Conflitos no entendimento do que seja o modelo multidimensional.

Os três Colégios que organizam as 49 Áreas de avaliação da CAPES, afirmam ainda, na Carta Aberta, que "todas as discussões que envolvam a definição de indicadores de avaliação dos programas de pós-graduação devem ser realizadas com a presença ou representação das instâncias científicas responsáveis por sua aplicação no SNPG, a saber, as Coordenações de áreas, os Colégios, o CTC-ES e o CS". Mostram também, na referida Carta Aberta, por meio de quadro comparativo, que o relatório da Comissão 2020 difere da "Proposta de Aprimoramento do Modelo de Avaliação da PG - Documento Final da Comissão Nacional de Acompanhamento do PNPG 2011-2020 - 10/10/2018". Ao final, reafirmam que

\begin{abstract}
a CAPES é um importante patrimônio brasileiro, que vem sendo construído ao longo das últimas seis décadas, contando com a participação ativa de toda a comunidade científica nacional e, por meio de seus mais de sete mil cursos, está presente em todas as regiões do país. Essa estrutura é responsável, em grande medida, pela detecção e solução de problemas, sinalizando os rumos para um progresso que considere as diversas necessidades de nossa gente: ciência, tecnologia, arte e cultura. Assim, a CAPES deve ser tratada com o devido respeito e nenhuma alteração em sua estrutura pode ser conduzida sem uma correta e ampla participação da nossa comunidade científica, de acordo com os ditames do regime democrático participativo, pautado na Constituição de 1988.
\end{abstract}

Ao que parece, no entanto, a Presidência da CAPES está disposta a implementar o que prevê o relatório da Comissão 2020, pois publicou a Portaria no 71, de 10 de junho de 2020, criando Comissão Especial destinada a propor e atualizar a classificação de áreas de avaliação no âmbito da Capes. No relatório da Comissão 2020 a previsão é reduzir de 49 para nove o número de áreas de avaliação, além de extinguir o Qualis Periódicos, uma vez que a avaliação irá basear-se em padrões e critérios propostos para as cinco dimensões do novo modelo: formação de pessoal; pesquisa; inovação e transferência de conhecimento; impacto na sociedade; e internacionalização.

A Comissão 2020 sugere ainda em seu relatório: a) manter a periodicidade do ciclo de avaliação de quatro anos; b) ter indicadores comuns pra todas as áreas de avaliação, não sendo permitido indicadores específicos; c) reduzir as áreas de avaliação para as nove grandes áreas de conhecimento; d) adotar uma única categoria de docentes do programa; e) favorecer a diversificação das estratégias de fomento; f) enfatizar o plano estratégico de cada programa e a autoavaliação; g) extinguir o Qualis Periódicos, passando a adotar métricas internacionais, a exemplo de índices de citação e impacto; h) manter a escala de notas de 1 a 7 , mas adotando uma escala de notas para cada dimensão, com patamar mínimo para cada dimensão para fins de permanência no sistema; i) considerar parâmetros próprios para admissão de novos PPGs no sistema, expresso por meio de resultado aprovado ou reprovado. 
Em sintonia com o relatório da Comissão 2020, em reunião on-line, realizada no dia 18 de junho de 2019, a equipe técnica de avaliação da pós-graduação da CAPES, ao apresentar o Tutorial de Dados Sucupira, informou que já no quadriênio 2017-2020 a metodologia de avaliação prevê $\bigcirc$ uso de indicadores bibliométricos externos/internacionais, visando a facilitar a padronização das regras. Serão usados para classificação da produção intelectual o percentil ou posição de cada periódico na respectiva área, especialmente por meio do Scorpus e Clarivete web of Science, podendo ser complementado pelo índice " $\mathrm{h}$ " do Google Scholar. O percentil é que vai determinar o extrato referência. Portanto, sugeriram que os docentes dos programas observem o fator de impacto do periódico antes de publicar. Isso significa que o extrato a ser obtido tem a ver com o impacto do periódico em bases internacionais, tais como Scorpus, Clarivete web of Science e índice " $h$ " do Google Scholar.

\section{CONSIDERAÇÕES FINAIS}

Pode-se inferir que o modelo multidimensional proposto pela Comissão 2020 sinaliza aos programas de pós-graduação que compõem o SNPG que, como nunca, a avaliação da pós-graduação nacional será orientada pela comparabilidade de performances internacionais, expressa em tabelas de classificação (rankings), e sua governança será baseada em indicadores, algo muito próximo dos três eixos da quarta versão do Programa FUTURE-SE, protocolado, em 02 de junho de 2020, como Projeto de Lei - PL 3076/2020:

Art. 4․ O Programa Future-se divide-se em três eixos: I - pesquisa, desenvolvimento tecnológico e inovação; II - empreendedorismo; e III - internacionalização.

Parágrafo único: As ações desenvolvidas no âmbito dos eixos do Programa Future-se têm $o$ propósito de contribuir para 0 aprimoramento dos esforços de pesquisa, desenvolvimento e inovação, observadas as políticas nacionais de ciência, tecnologia e inovação e as prioridades temáticas definidas pelo Ministério da Ciência, Tecnologia, Inovações e Comunicações (BRASIL, 03 jun. 2020).

Neste sentido, pode-se deduzir que formação de pessoal em nível de graduação e pós-graduação, pesquisa (produção científica), inovação e extensão, estão intimamente conectados. Como uma evidência, conforme dados compilados pela Clarivate Analytics a pedido da CAPES, vale ressaltar que $95 \%$ da produção científica brasileira originam-se em universidades públicas (federais e estaduais), cujos autores, em grande medida, são docentes credenciados em programas de Pós-Graduação (MOURA, 16 abr. 2019). Para testemunhar essa constatação, Luiz Davidovich, presidente da Associação Brasileira de Ciência ( $A B C)$, em entrevista à autora de artigo de divulgação publicado pela Universidade Federal de São Paulo (Unifesp), afirma que "mais de $95 \%$ das publicações referem-se às universidades públicas, federais e estaduais" (MOURA, 16 abr. 2019). 
Entretanto, a maior "inovação" no campo da educação superior no Brasil, especialmente a respeito do SNPG, pode vir a ser o efeito perverso da combinação entre a promoção de tabelas de classificação (rankings), como o modelo multidimensional, inspirado no U-Multirank em operação na Europa, e o estabelecimento de Contratos de Resultados, como condição de obtenção de benefícios e financiamentos, entre autoridades federais (União/MEC) e Instituições de Federais de Educação Superior (IFES), regulado por indicadores de performance, conforme previsto no art. $3^{\circ}$., do PL 3076/2020, referente à quarta versão do Programa FUTURE-SE:

Art. $3^{\circ}$ Para fins do disposto nesta Lei, considera-se: I - contrato de resultado - instrumento jurídico celebrado entre universidades ou institutos federais e a União, por intermédio do Ministério da Educação, caracterizado por consensualidade, objetividade, responsabilidade e transparência, com a finalidade de estabelecer indicadores de resultado para a contratada, como contrapartida da concessão de benefícios por resultado (BRASIL, 02 jun. 2020).

Enfim, diante dos dados expostos e do contido no Relatório da Comissão Especial de Acompanhamento do PNPG 2011-2020, que apresentou uma "Proposta de Aprimoramento da Avaliação da Pós-Graduação Brasileira para o Quadriênio 2021-2024", quer parecer que a ideia de governança por indicadores e manuais de boas práticas, também chamada de administração por Benchmarking (AZEVEDO, 2016b), passa a ser manifestamente a ferramenta de regulação do SNPG, dos sistemas federal e estaduais de educação e do diversificado conjunto de instituições, organizações, plataformas e laboratórios de produção de Ciência, Tecnologia e Inovação no Brasil.

Nesse contexto de alterações, no entanto, é preciso considerar que a estruturação e consolidação da pós-graduação no Brasil só foi possível a partir do momento em que ela se tornou uma política de Estado, alicerçada em dois pilares: avaliação dos programas/cursos em articulação com as comunidades científicas das diferentes áreas de conhecimento e financiamento permanente para manutenção e expansão do sistema. O resultado disso tem sido a qualidade de padrão internacional da pós-graduação e da pesquisa no país, cada vez mais internacionalizada, e a expansão permanente do sistema e dos indicadores de produção do conhecimento. Mas vivemos uma conjuntura no Brasil de questionamento à ciência e de redução do papel regulador e financiador do Estado. Além dos dilemas existentes, estamos agora frente a desafios que apontam para uma mudança significativa nas finalidades e nos rumos da pós-graduação brasileira, que tende a ocorrer nos moldes apresentados caso não se estabeleça um amplo diálogo com as áreas no interior da CAPES e com as entidades científicas das diferentes áreas de conhecimento que sempre estiveram presentes e foram protagonistas na constituição do atual sistema de pós-graduação. 


\title{
POSTGRADUATE PROGRAMS AND KNOWLEDGE PRODUCTION IN BRAZIL: OVERVIEW, CHALLENGES AND PERSPECTIVES
}

\begin{abstract}
The purpose of this article is to analyze the situation of knowledge production and postgraduate programs in Brazil, considering the impacts resulting from the so-called globalization of the knowledge economy. To this end, national and international data on the production and dissemination of knowledge are examined. Thus, the text highlights the new logic of regulation and evaluation of postgraduate programs, as well as points out that, beyond the existing economic, political and social dilemmas, there are challenges related to significant changes in Brazilian postgraduate studies. The text also calls attention to new trends in the evaluation of postgraduate studies in Brazil and to the necessary dialogue between CAPES knowledge areas coordinators, CAPES regulatory bodies and scientific entities that have always been present and were protagonists in the constitution of the current system graduate programs in Brazil.
\end{abstract}

KEYWORDS: Postgraduate programs. Knowledge production. Postgraduate Evaluation. Knowledge economy.

\section{PROGRAMAS DE POSTGRADO Y PRODUCCIÓN DE CONOCIMIENTOS EN BRASIL: VISIÓN GENERAL, RETOS Y PERSPECTIVAS}

RESUMEN: El propósito de este artículo es analizar la situación de la producción de conocimiento y los programas de posgrado en Brasil, considerando los impactos resultantes de la llamada globalización de la economía del conocimiento. Con este fin, se examinan los datos nacionales e internacionales sobre la producción y difusión del conocimiento. Por lo tanto, el texto destaca la nueva lógica de regulación y evaluación de los programas de posgrado, y señala que, más allá de los dilemas económicos, políticos y sociales existentes, existen desafíos relacionados con cambios significativos en los estudios de posgrado brasileños. El texto también llama la atención sobre las nuevas tendencias en la evaluación de los estudios de posgrado en Brasil y el diálogo necesario entre los coordinadores de áreas de conocimiento de CAPES, los organismos reguladores de CAPES y las entidades científicas que siempre han estado presentes y fueron protagonistas en la constitución de los programas de posgrado del sistema actual. en Brasil.

PALABRAS CLAVE: Estudios de posgrado. Producción de conocimiento. Evaluación de posgrado. economía del conocimiento.

\section{NOTAS}

1) A legislação específica da CAPES voltada à pós-graduação pode ser consultada em: https://capes.gov.br/avaliacao/sobre-a-avaliacao/legislacao-especifica.

2) A Comissão Especial de Acompanhamento do PNPG 2011-2020, representando diversos segmentos do campo acadêmico nacional, foi composta pelo seguintes nomes: Jorge Luís Nicolas Audy - Pontifícia Universidade Católica (PUC/RS), Presidente da Comissão; Adalberto Luís Val Instituto Nacional de Pesquisas da Amazônia (INPA); Adalberto Grassi Carvalho - Coordenação de 
OLIVEIRA, J. F. de; AZEVEDO, M. L. N. de

Aperfeiçoamento de Pessoal de Nível Superior (CAPES); Carlos Eduardo Pereira - Universidade Federal do Rio Grande do Sul (UFRGS); Guilherme Ary Plonski - Universidade de São Paulo (USP); José Fernandes de Lima - Universidade Federal de Sergipe (UFS); Lúcia Galvão de Albuquerque Universidade Estadual de São Paulo (UNESP); Luiz Roberto Liza Curi - Conselho Nacional de Educação (CNE); Márcio de Castro Silva Filho - Fórum de Pró-Reitores de Pesquisa e Pós-Graduação (FOPROP); Nei Yoshihiro Soma - Instituto Tecnológico da Aeronáutica (ITA); Rui Otávio Bernardes de Andrade - Universidade Estácio de Sá - (UNESA); Santuza Maria Ribeiro Teixeira - Universidade Federal de Minas Gerais (UFMG).

3) ORIGINAL: "Based on the results of a feasibility study covering 150 universities which was carried out in 2010/11, the European Commission decided to fund the implementation of U-Multirank" 4) ORIGINAL: "U-Multirank is a multidimensional, user-driven approach to international ranking of higher education institutions. It compares the performances of higher education institutions - in short: universities - in the five dimensions of university activity: (1) teaching and learning, (2) research, (3) knowledge transfer, (4) international orientation and (5) regional engagement".

5) As informações da ficha de avaliação foram sistematizadas e disponibilizadas pela Coordenação de área de Educação da CAPES, representada pelo Coordenador de área de Educação, Prof. Dr. Robert Evan Verhine, Coordenador Adjunto, prof. Dr. Ângelo Ricardo de Souza, e Coordenador Adjunto para Programas Profissionais, Prof. Dr. Luiz de Sousa Junior, em Seminário da CAPES no meio do quadriênio e em apresentações e discussões públicas em eventos promovidos pela Associação Individual Associação Institucional (ANPEd) e PPGEs.

6) De acordo com a Coordenação de Área da Educação e segundo o que consta na ficha de avaliação, a coautoria entre docentes do mesmo PPG conta somente para um dos professores credenciados. No máximo um dos produtos bibliográficos pode ser publicado em revistas científicas vinculadas ao PPG, ou Faculdade de Educação ou equivalente, ou editora universitária vinculada à IES.

\section{REFERÊNCIAS}

ALVES, M. F.; OLIVEIRA, J. F. Pós-Graduação no Brasil: do Regime Militar aos dias atuais. RBPAE - v. 30, n. 2, p. 351-376, mai./ago. 2014. Disponível em: https://seer.ufrgs.br/rbpae/article/view/53680/33095.

AZEVEDO, M. L. N. A economia baseada no "cercamento" do conhecimento: globalização, educação e mercadorias fictícias. In: CHAVES, Vera Jacob; SILVA JÚNIOR, João dos Reis; CATANI, Afrânio Mendes (Orgs.). A Universidade brasileira e o PNE: instrumentalização e mercantilização educacionais. São Paulo: Xamã, 2013. p. 95-112.

AZEVEDO, M. L. N. O Novo Regime Fiscal: a retórica da intransigência, o constrangimento da oferta de bens públicos e o comprometimento do PNE 2014-2024. Tópicos

Educacionais, v. 1, p. 234-258, 2016 a.

AZEVEDO, M. L. N. Educação e Benchmarking: meta-regulação e coordenação de políticas baseadas em indicadores e nas chamadas 'boas-práticas'. Anais do XXIV Seminário Nacional Universitas/Br, 18 a 20 de Maio de 2016, ISSN 2446-61232016. Universidade Estadual de Maringá: 2016b. 
AZEVEDO, M. L. N.; OLIVEIRA, J. F.; CATANI, A.M. O Sistema Nacional de Pós-graduação (SNPG) e o Plano Nacional de Educação (PNE 2014-2024): regulação, avaliação e financiamento. RBPAE - Periódico científico editado pela ANPAE, [S.I.], v. 32, n. 3, p. 783 803, dez. 2016. ISSN 2447-4193. Disponível em:

https://seer.ufrgs.br/rbpae/article/view/68576/39684.

BALL, S. J.; BOWE, R. Subject departments and the 'implementation' of National Curriculum policy: an overview of the issues. Journal of Curriculum Studies. Pages 97115, 09 Jul 1992 doi.org/10.1080/0022027920240201.

BRASIL. Coordenação de Aperfeiçoamento de Pessoal de Nível Superior (CAPES). CAPES apresenta à academia modelo de avaliação multidimensional. Portal da CAPES. Publicado: 02 out 2019. Extraído de https://www.capes.gov.br/36-noticias/9873-capesapresenta-a-academia-modelo-de-avaliacao-multidimensional.

BRASIL. Coordenação de Aperfeiçoamento de Pessoal de Nível Superior (CAPES). Proposta de Aprimoramento da Avaliação da Pós-Graduação Brasileira para o Quadriênio 2021-2024 - Modelo Multidimensional. Relatório da Comissão Especial de Acompanhamento do PNPG 2011-2020. Brasília: CAPES, fev. 2020.

BRASIL. Coordenação de Aperfeiçoamento de Pessoal de Nível Superior (CAPES). Ficha de Avaliação - 38 Educação - 2017/2020. Coordenador da Área: Robert Evan Verhine, Coordenador Adjunto de Programas Acadêmicos: Ângelo Ricardo de Souza, Coordenador de Programas Profissionais: Luiz de Sousa Junior. Brasília: CAPES, 20172020.

BRASIL. Projeto de Lei $n^{\circ}$ 3076/2020 - Programa Universidades e Institutos Empreendedores e Inovadores - FUTURE-SE (4a. versão, de 02 jun 2020). Extraído de https://www.camara.leg.br/proposicoesWeb/prop_mostrarintegra?codteor=1900012. Acesso em 07 jun 2020.

BRASIL. Instituto Nacional de Estudos e Pesquisas Educacionais Anísio Teixeira (Inep). Relatório do $2^{\circ}$ ciclo de monitoramento das metas do Plano Nacional de Educação 2018. - Brasília, DF: Inep, 2018.

HOWMUCH. Understanding Money. 2017. Extraído de https://howmuch.net/

INSTITUTO NACIONAL DA PROPRIEDADE INDUSTRIAL (INPI). Boletim mensal de propriedade industrial: estatísticas preliminares. Instituto Nacional da Propriedade Industrial (INPI). Presidência. Diretoria Executiva. Assessoria de Assuntos Econômicos (AECON) - vol. 1, n. 1 (2016) - Rio de Janeiro: INPI, 2020. Disponível em: http://www.inpi.gov.br/sobre/estatisticas. Acesso em: 21 mai. 2020. 
LIMA, L. C. O paradigma da educação contábil: políticas educativas e perspectivas gerencialistas no Ensino Superior em Portugal. Revista Brasileira de Educação, no 4, 1997, p. 43-59.

MARGINSON, S. Global cooperation, national competition and social inequality in the World-Class University (WCU) sector. Working paper $n^{\circ} 34$, April 2018. Centre for Global Higher Education - funded by the Economic and Social Research Council (ESRC) and the Higher Education Funding Council for England (HEFCE) and based at the UCL Institute of Education). 2018.

MOURA, M. Universidades públicas realizam mais de $\mathbf{9 5 \%}$ da ciência no Brasil. São Paulo: UNIFESP, 16 abr. 2019. Disponível em: https://www.unifesp.br/noticiasanteriores/item/3799-universidades-publicas-realizam-mais-de-95-da-ciencia-no-brasil. Acesso em: 14 jun. 2020.

OLIVEIRA, J. F.; AMARAL, Nelson C. As transições políticas no brasil e seus efeitos na educação brasileira. 2020 (mimeo).

OLIVEIRA, J. F.; LIMA, D. C. B. P. Pós-graduação e educação à distancia: novos fins, natureza e modus operandi em construção. In: CASTRO, A. et. all (Orgs). Educação Superior em países e regiões de língua portuguesa: desafios em tempo de crise. 1ed. Lisboa: ED Educa, 2018, v. 1, p. 215-244.

OLIVEIRA, J. F. A Pós-Graduação e a pesquisa no Brasil: processos de regulação e de reconfiguração da formação e da produção do trabalho acadêmico. Práxis Educativa, Ponta Grossa, v. 10, n. 2, p. 343-363, jul./dez. 2015. Disponível em:

http://revistas2.uepg.br/ojs_new/index.php/praxiseducativa

SAVIANI, D. O dilema produtividade-qualidade na Pós-Graduação. Nuances: estudos sobre educação, Presidente Prudente, ano XVII, v. 17, p. 35-50, jan./dez. 2010.

SOUZA, Â. R. Avaliação da Pós-Graduação em Educação [mensagem pessoal]. Mensagem recebida por mlnazevedo@uem.br, enviado por angelosou@gmail.com em 17 mar. 2020.

U-MULTIRANK. U-Multirank Project. Extraído de https://www.umultirank.org/about/umultirank/the-project/. Acesso em 15 jun 2020.

CLARIVATE ANALYTICS. Web of Science Group. A Pesquisa no Brasil: Promovendo a excelência - Análise preparada para a CAPES pelo Grupo Web of Science. Clarivate Analytics, 2019. Disponível em:

https://www.sbponline.org.br/arquivos/Promovendo_a_excele\%CC\%82ncia.pdf 
João Ferreira de Oliveira: Possui graduação em Pedagogia pela Universidade Federal de Goiás - UFG (1989), Mestrado em Educação pela UFG (1994), Doutorado em Educação pela Universidade de São Paulo - USP (2000) e Pós-doutorado em Educação pela USP (2010 e 2016). É Professor Titular da UFG.

Orcid: https://orcid.org/0000-0002-4135-6340

E-mail: joao.jferreira@gmail.com

MÁrio Luiz Neves de Azevedo: Professor Titular da Universidade Estadual de Maringá (UEM). Mestre em Educação pela Universidade Federal de São Carlos (UFSCar). Doutor em Educação pela FE da USP, com estágio de pesquisa (bolsa-sanduíche/CAPES) no Institut National de Recherche Pédagogique (INRP-França). Pós-doutorado na Universidade de Bristol-Inglaterra (2011). Pesquisador visitante na Universidade de Cambridge-Inglaterra (2018-2019) com bolsa PVE-CAPES.

Orcid: https://orcid.org/0000-0003-0563-5817

E-mail:mlnazevedo@uem.br

Este periódico utiliza a licença Creative Commons Attribution 3.0, para periódicos de acesso aberto (Open Archives Iniciative - OAI). 